\title{
FACTIBILIDAD TÉCNICA DE UN HORNO SOLAR PARA LA COCCIÓN DE LADRILLOS EN LA CIUDAD DE VALLEDUPAR (COLOMBIA)
}

\section{TECHNICAL FEASIBILITY OF A SOLAR OVEN FOR THE COOKING OF BRICKS IN THE CITY OF VALLEDUPAR (COLOMBIA)}

\author{
Ing. Álvaro Doria Álvarez*, Msc. Ing. Javier Orozco Ospino*, PhD. Carlos Arrieta* \\ *Fundación Universitaria del Área Andina, Facultad de Ingeniería y Ciencias Básicas \\ Trans 22 Bis \# 4 -105, Sector Callejas, Valledupar, Cesar, Colombia. \\ Teléfono: $(+57)(5) 5897879$ \\ E-mail: alvarodorialvarez@gmail.com, jaorozco@areandina.edu.co, \\ carrieta9@areandina.edu.co
}

Resumen: En el marco de la mejora de materiales, no solo se tiene en cuenta mejorar sus propiedades o capacidades para cierta aplicación, sino también la reducción de su huella ecológica, en la cual intervienen factores como el transporte e incluso la fabricación, que en general son procesos con un gran impacto ambiental. En este trabajo, en especial, se aborda la problemática ambiental derivada de la fabricación de los ladrillos en la región de Valledupar (Colombia), el cual en general es un proceso artesanal. Razón por la que este trabajo presenta un estudio de factibilidad de un horno solar que ayude a mitigar las emisiones nocivas producidas por el gremio ladrillero. Para ello se presentan las metodologías de cálculo para el diseño de hornos y así estimar el consumo energético de un horno Pampa típico en la región, el cual es comparado con la energía aprovechada de colectores solares dispuestos en forma parabólica con un punto focal como concentrador de la energía, determinando que el potencial de energía calórica concentrada del sol es mayor que la energía requerida por un horno Pampa tradicional lo que indica que la propuesta del horno solar es factible técnicamente.

Palabras clave: horno solar, heliostato, ladrillo.

\begin{abstract}
In the framework of the improvement of materials, not only takes into account improve their properties or capabilities for a certain application, but also the reduction of their ecological footprint, which involves factors such as transport and even manufacturing, which in general are processes with a great environmental impact. In this work, in particular, the environmental problems derived from the manufacture of bricks in the region of Valledupar (Colombia), which in general is an artisanal process, are addressed. Reason why this work presents a feasibility study of a solar furnace that helps to mitigate the harmful emissions produced by the brickmaking guild. For this purpose, the calculation methodologies for the design of furnaces are presented, thus estimating the energy consumption of a typical Pampa furnace in the region, which is compared with the energy taken from solar collectors arranged in a parabolic shape with a focal point as a concentrator. energy, determining that the concentrated heat energy potential of the sun is greater than the energy required by a traditional Pampa furnace, which indicates that the solar oven proposal is technically feasible.
\end{abstract}

Keywords: solar oven, heliostat, brick. 


\section{INTRODUCCIÓN}

En la ciudad de Valledupar y sus alrededores, la mayor parte de la producción de ladrillos se realiza de forma artesanal, con procesos ineficientes que no van acordes con el avance del gremio de la construcción en otras ciudades de la región Caribe Colombiana. Estudios recientes muestran que este proceso presenta una serie de problemas particulares, partiendo de las altas tasas de combustible necesarias para su funcionamiento, que, si bien pueden ser petróleo, gas o carbón, el empleado comúnmente en Valledupar es la madera (Luján \& Guzmán, 2015). Es sabido que la cantidad y calidad del combustible está estrechamente relacionado con los rendimientos de los hornos, sin desconocer el impacto ambiental que causa la quema de estos, y la huella ecológica que dejan. Especialmente los hornos pampas usados de manera predominante en la región (Cesar, Gobernacion del, 2013). Por este motivo es de gran interés el estudio de nuevos modelos de producción que permitan introducir alternativas en el proceso de fabricación de ladrillo. (JS Molina, JFG Díaz. 2013).

En este contexto es evidente la necesidad de innovar e implementar en el departamento del Cesar nuevas técnicas, que a través de un proceso de fabricación limpia, obtengan un producto con iguales o mejores propiedades, ya que la mayoría de los ladrillos elaborados en el municipio requieren de prácticas que afectan la integridad del medio ambiente (Galindo Rodriguez, 2015), de los operarios y las comunidades cercanas a los procesos (Cotes, Núñez, \& Sabogal, 2012). En este trabajo se busca en el horno solar una alternativa para la producción de ladrillos que sea técnicamente viable, con impacto ambiental mucho menor, y que, al mismo tiempo, genere competitividad e innovación en la región para la fabricación de ladrillos. (GG Gómez, EE Blanco.2018).

\section{la cocción}

En general los ladrillos empleados en construcción requieren para su proceso industrial una serie de transformaciones donde la más importante es el proceso químico que sucede en la quema, ya que es debido a este que se logra obtener una pieza resistente, con buena textura, porosidad, y sonido metálico característicos de los cerámicos; estas reacciones químicas comienzan en la medida que el ladrillo gana temperatura y finalizando en el rango de temperatura comprendida entre $950{ }^{\circ} \mathrm{C}$ y 1100 ${ }^{\circ} \mathrm{C}$, a temperaturas superiores se producen la fusión de parte de sus compuestos aumentando las pérdida del ladrillo. (Casado Piñeiro) (Garcia, 2014)

\section{Radiación solar en la ciudad de Valledupar}

La ciudad de Valledupar cuenta con importantes recursos renovables de los cuales se destaca la radiación solar, la cual permanece más o menos constante a lo largo del año, como se presenta en la Ilustración 1 y en la Tabla 1 .

Ilustración 1. Mapa de radiación solar

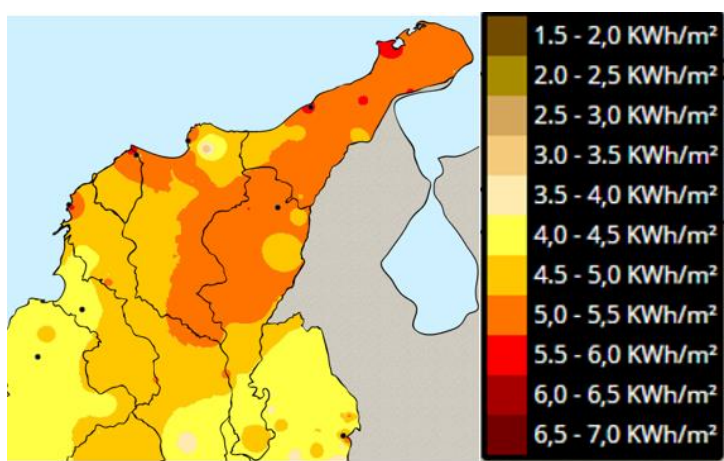

(IDEAM, 2017)

Tabla 1. Radiación solar diaria horizontal

\begin{tabular}{|lcc|} 
& & \\
& & \\
& & \\
\hline Enero & 23.8 & 5.46 \\
febrero & 24.9 & 5.53 \\
Marzo & 26 & 5.74 \\
Abril & 25.1 & 5.39 \\
Mayo & 24.8 & 5.17 \\
Junio & 24.7 & 5.29 \\
julio & 24.6 & 5.47 \\
Agosto & 24.8 & 5.28 \\
Septiembre & 24.5 & 5.08 \\
Octubre & 24.2 & 4.77 \\
Noviembre & 23.8 & 4.82 \\
Diciembre & 23.4 & 5 \\
Anual & $\mathbf{2 4 . 6}$ & $\mathbf{5 . 2 5}$ \\
\hline
\end{tabular}

(NASA, 2017) 


\section{Colector solar.}

Es pertinente tener en cuenta algunos datos importantes en consideración, como lo es la razón de concentración superficial, que se define como el cociente del área de apertura del concentrador entre el área de absorción del receptor, explicado, en otros términos, es cuanta energía puede acumular en un punto específico. Para efectos de aclaración se presenta en la tabla 2 los diferentes tipos de concentradores existentes, comparando su razón de concentración con la temperatura que estos pueden alcanzar.

\section{Tabla 2. clasificación de colectores solares según su factor de concentración}

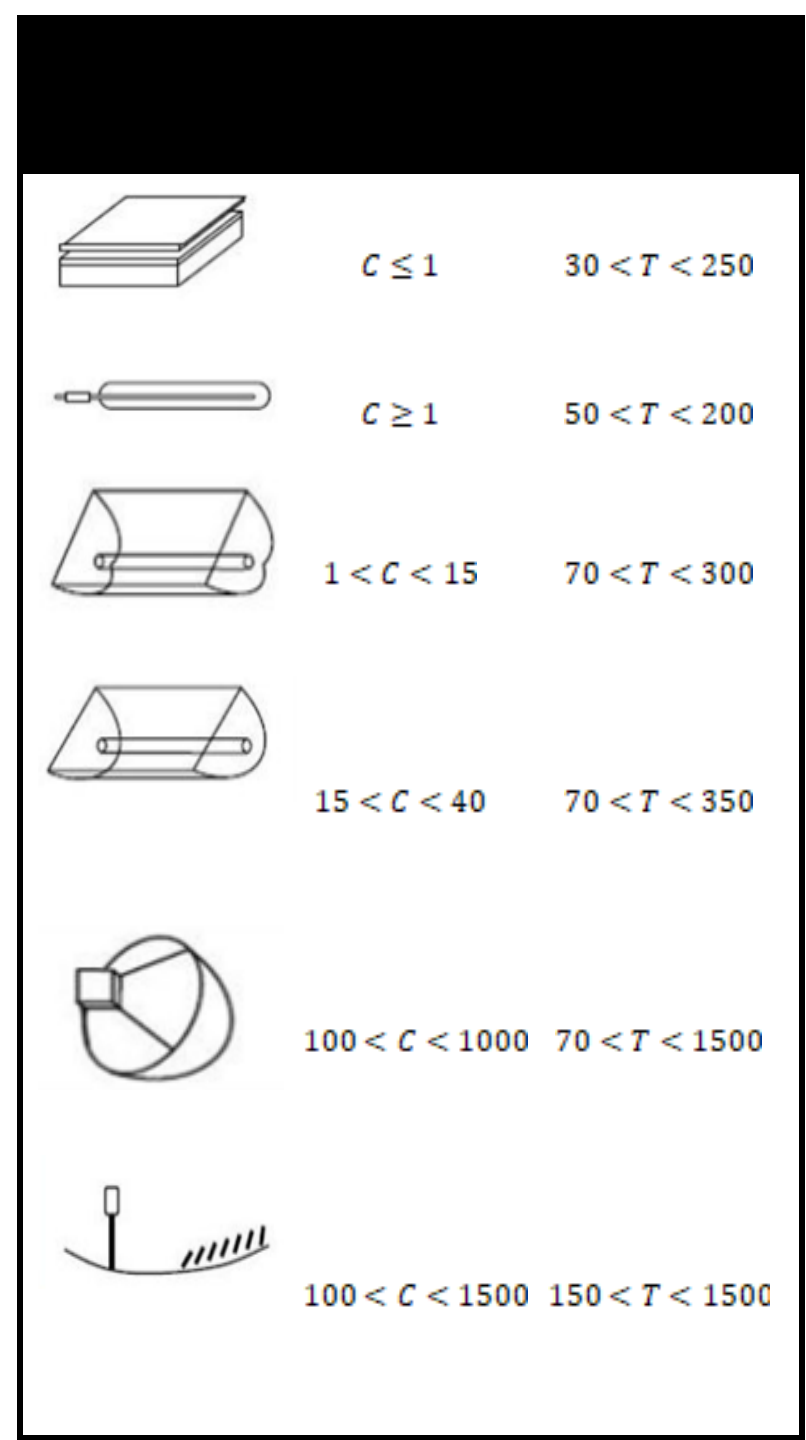

(Cepeda, A. \& Otáñez, G, 2007)

\section{MATERIALES Y MÉTODO}

Para empezar el estudio de factibilidad técnica de un horno ladrillero solar se partió por comprobar los requerimientos energéticos de un horno convencional. Se realizó un balance de energía, en el que fue necesario definir lo mecanismos de transferencia de calor presentes en el proceso. Todo esto siguiendo lo reportado en la norma ISO 50001. (ISO 50001, 2011)

El horno analizado fue horno pampa de tiro vertical, cuyas características geométricas principales se presentan en la ilustración 2 y 3

Ilustración 2. Plano en planta de horno ladrillero (medidas en metros)

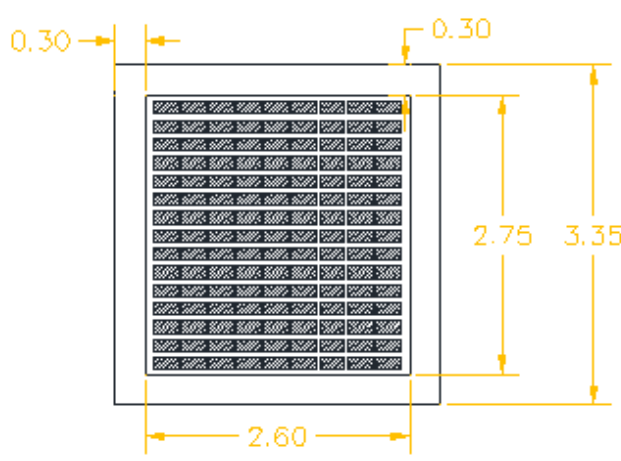

Ilustración 3. Plano de corte del horno ladrillero (medidas en metros)

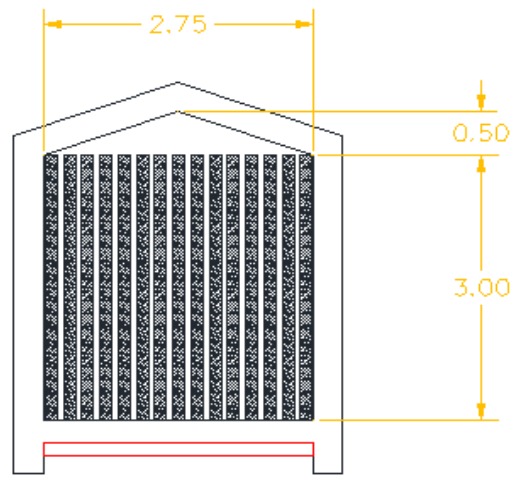

Algunas propiedades importantes del horno, que fueron utilizadas para la implementación de las metodologías de cálculo reportadas en la norma ISO 50001, es que los muros son de arcilla refractaria con un espesor de $0.30 \mathrm{~m}$, y tiene una capacidad de quema 2700 ladrillos en los que se quema un ladrillo de perforación horizontal (nombre comercial H12) comúnmente usado en construcciones de la ciudad. 
Los ladrillos cuentan con una dimensión $0.15 \mathrm{~m} *$ $0.12 \mathrm{~m} * 0.25 \mathrm{~m}$, un área de la sección transversal $0.004 \mathrm{~m} 2$ y un volumen del ladrillo $0.001 \mathrm{~m} 3$, como se muestra en la ilustración 4.

Ilustración 4. Sección transversal de ladrillo (medidas en metros)

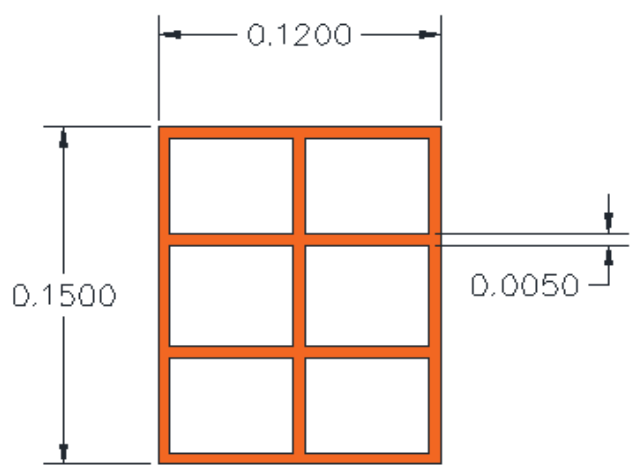

Una vez calculado el consumo de energía necesario para una quema de ladrillos, se escoge un colector que sea capaz de genera esta energía y la temperatura necesaria para el proceso, por lo que hay que tener en cuenta el tipo de colector empleado y las perdidas asociadas a este, para obtener una energía útil que debe ser mayor a la energía consumida por la cocción.

Todo esto es pertinente para poder calcular el rendimiento energético del colector con factor $100<C<1500$ como se muestra en la tabla 3 .

Con respecto al sistema se empleó espejos formando un pequeño heliostato como se muestra en la ilustración 5. (POZOS SUÁREZ, 2010)

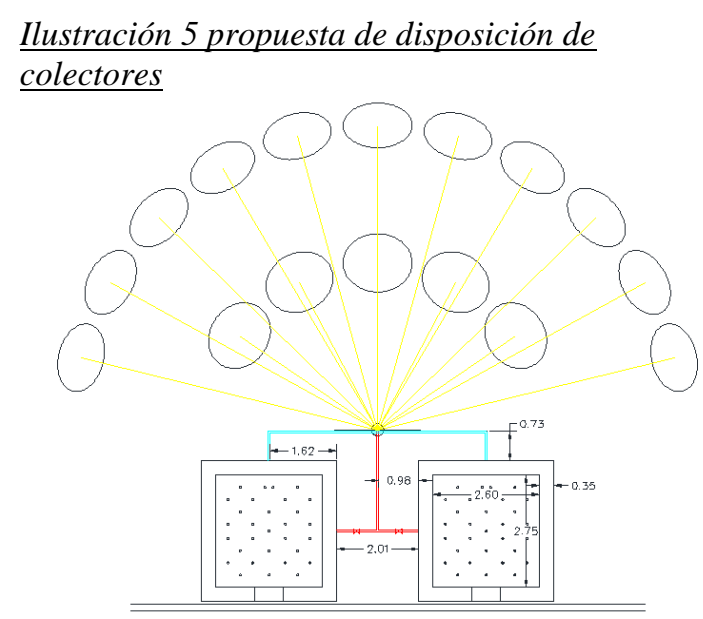

Universidad de Pamplona I. I. D. T. A.
Tabla 3. Ecuaciones de rendimiento energético de los colectores

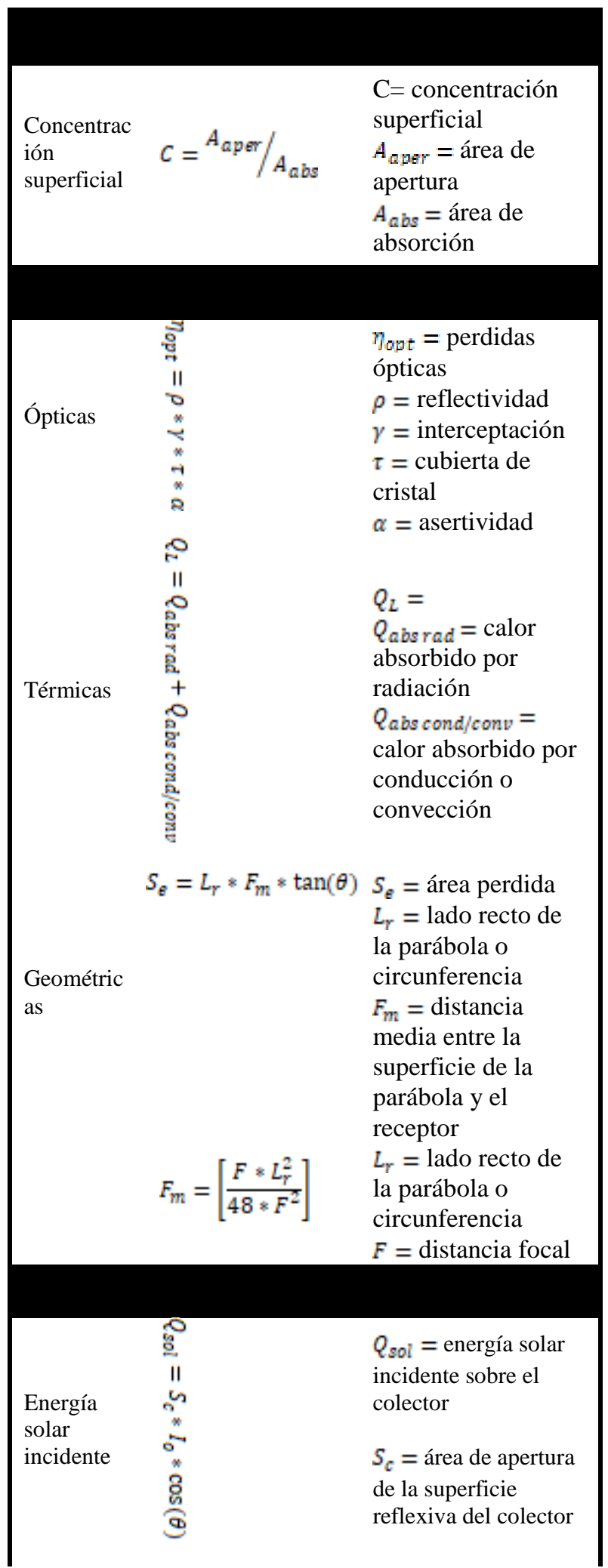




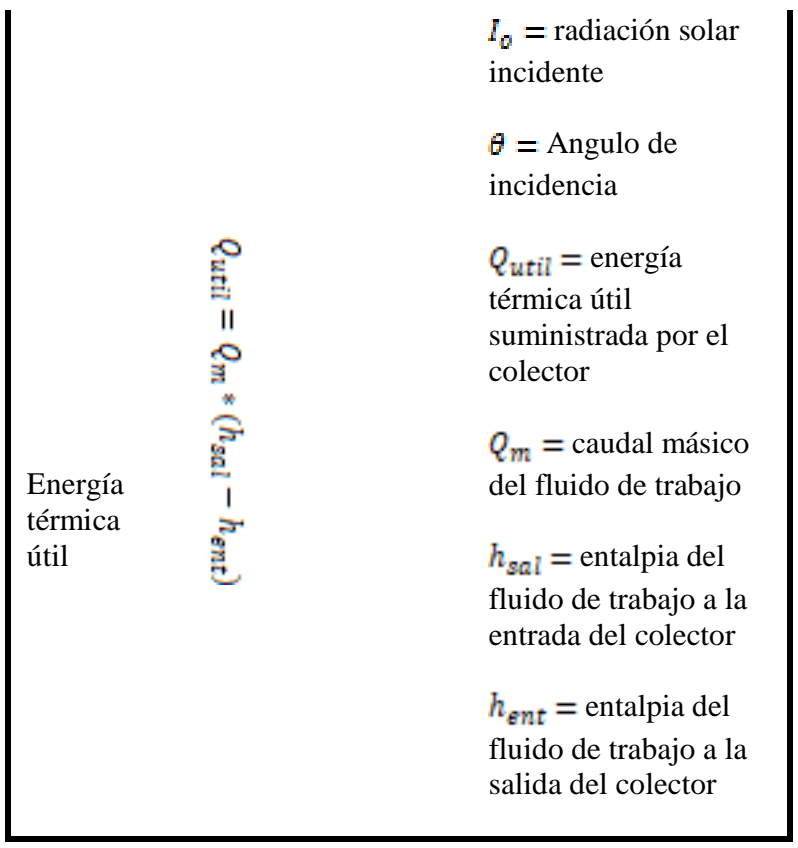

RESULTADOS Y DISCUSIÓN

El primer paso para afrontar es cuantificar el consumo energético estimado del horno, como se muestra en el gráfico 1. Gráfico 1. Energía consumida por el horno
$\underline{\text { unidades en kilo Joules) }}$

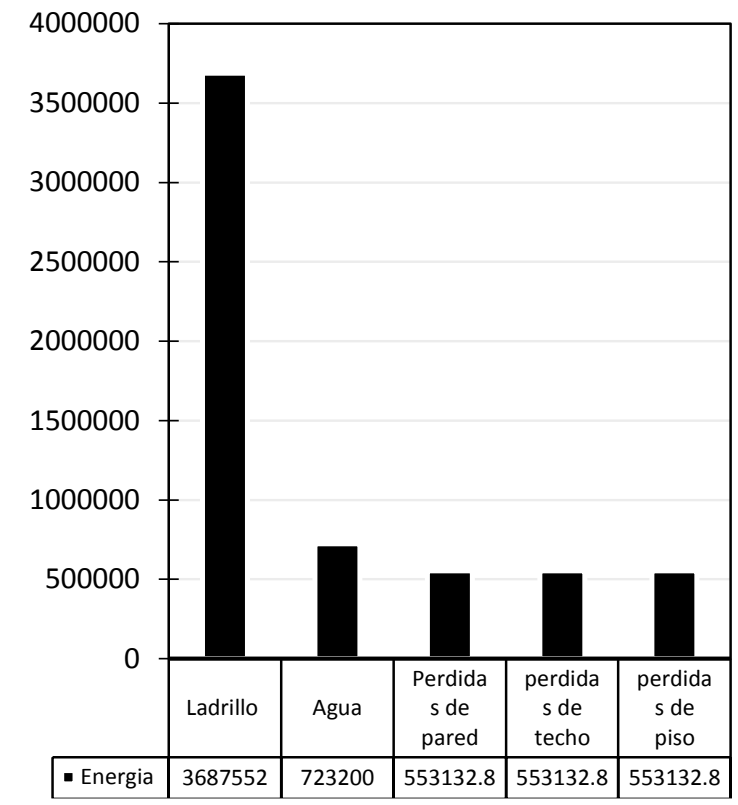

Consumo energético de una quema de ladrillos corresponde a 603,378 watt de potencia.
Estos cálculos se basaron en las dimensiones del horno descrito; las pérdidas de energía de los elementos constructivos de techos, pisos y paredes se estimaron del 9\% del total de la energía consumida por el horno (Casado Piñeiro).

Ya conocido la energía consumida por el sistema expuestas en el grafico 1, se procedió a calcular la captación de energía de los colectores propuestos obteniendo la energía concentrada por los colectores especificada en la tabla 4. Cabe resaltar que no se consideró las pérdidas de atmosfera, ni las perdidas por difusión por nubosidad

\section{Tabla 4. Calculo de energía suministrada}

\begin{tabular}{|c|c|c|c|}
\hline & valor & unidad & pción \\
\hline $\mathbf{Q}$ & 29987,6072 & $\mathrm{~W}$ & energía \\
\hline $\begin{array}{l}\text { sola } \\
\mathbf{r}\end{array}$ & 8 & & proporcionada por \\
\hline Sc & 35,891 & $\mathrm{~m} 2$ & $\begin{array}{l}\text { área de apertura de } \\
\text { la superficie }\end{array}$ \\
\hline Io & 928,357 & $\mathrm{~W} / \mathrm{m} 2$ & $\begin{array}{l}\text { radiación solar } \\
\text { incidente }\end{array}$ \\
\hline $\begin{array}{l}\cos \\
(\Phi)\end{array}$ & 0,9 & & $\begin{array}{l}\text { coseno del ángulo } \\
\text { de incidencia }\end{array}$ \\
\hline & valor & unidad & descripción \\
\hline $\mathrm{Sc}$ & 35,891 & $\mathrm{~m} 2$ & $\begin{array}{l}\text { área de apertura de } \\
\text { la superficie } \\
\text { reflexiva }\end{array}$ \\
\hline $\mathrm{Ne}$ & 16 & & número de espejos \\
\hline $\mathrm{r}$ & 0,845 & $\mathrm{~m}$ & $\begin{array}{l}\text { radio de los } \\
\text { espejos }\end{array}$ \\
\hline & valor & unidad & descripción \\
\hline Io & 928,357 & $\mathrm{~W} / \mathrm{m} 2$ & $\begin{array}{l}\text { radiación solar } \\
\text { incidente }\end{array}$ \\
\hline
\end{tabular}

En todos los resultados presentados en la Tabla 4 la duración del proceso se asumió de 5.6 horas de acuerdo con las horas sol pico de Valledupar como se observa en la tabla 1.

Observando la potencia necesaria por el sistema y la generada por los colectores $(603,378 \mathrm{~W}<$ $29987,607 \mathrm{~W}$ ) por lo tanto es técnicamente factible la construcción de un horno de estas características 


\section{CONCLUSIONES}

Es técnicamente factible el diseño y construcción de un horno ladrillero solar de alimentado por heliostatos (espejos concentradores) y con la energía calorífica generada por estos, calentar una cámara de cocción ladrillera hasta la temperatura requerida por los ladrillos, aunque también hay que tener en cuenta que bajo condiciones reales existen variables que tienen que ser ajustadas al modelo como de difusión condiciones atmosférica y nubosidad,

Si bien es viable, aún falta desarrollar sistemas optimizados de este, es decir, falta hacer análisis mas detallados sobre los intercambiadores de energía en el receptor (sitio en el que se concentra la radiación) y su transmisión a la cámara de cocción.

\section{REFERENCIAS}

Casado Piñeiro, M. (s.f.). 2010. ELABORACIÓN DE LÍMITES MÁXIMOS PERMISIBLES DE EMISIONES PARA LA INDUSTRIA LADRILLERA.

Cepeda, A., S., \& Otáñez, G, D. (2007). Desarrollo de objetos utilitarios en cerámica otorgándoles un valor agregado a través de nuevas propuestas tecnológicas y de diseño.

http://dspace.udla.edu.ec/handle/33000/1 923.

Cesar, Gobernacion del. (2013). Estudio de factibilidad al proceso de reconversion de la produccion en la industria del ladrillo en los municipios de Valledupar y San Diego del departamento del Cesar. Valledupar.

Cotes, D., Núñez, D., \& Sabogal, L. (2012). Determinación de la calidad de los ladrillos a partir de la estimación de la resistencia última en las canteras de Valencia de Jesús, Las Casitas y El Cielo, en el municipio de Valledupar, Cesar. Revista agunkuya, Vol 2(1).

Galindo Rodriguez, M. (2015). Diseño de fábrica ladrillera potencializando procesos productivos, ambiente laboral e impacto medioambiental. PONTIFICIA UNIVERSIDAD JAVERIANA.

Garcia, S. M. (2014). Evaluación del proceso productivo de la planta industrial
LADRILLERA TERRAFORTE, ubicada en el sector de Calacalí en el periodo 2012-2013 (Thesis). Universidad Internacional SEK.

GG Gómez, EE Blanco. (2018), Curva De Cocción De La Arcilla En La Ladrillera El Recreo Revista Colombiana de Tecnologías de Avanzada ISSN: 1692-7257

IDEAM. (8 de enero de 2017). Atlas Interactivo Radiación . Obtenido de Atlas Interactivo - Radiación : http://atlas.ideam.gov.co/visorAtlasRadiac ion.html

ISO 50001. (2011). sistema de gestión de energía en una organización.

JS Molina, JFG Díaz. (2013), Implementación de un sistema tipo Scada para mejorar los procesos de secado y cocción de la ladrillera sigma Ltda. Revista Colombiana de Tecnologías de Avanzada ISSN: 16927257

Luján, M., \& Guzmán, D. (2015). Diseño, Construcción y Evaluación de un Horno (MK3) para la Cocción de Ladrillos Artesanales. Departamento de Ciencias Exactas e Ingeniería, Universidad Católica Boliviana San Pablo.

Meythaler Naranjo, E. (2014). Termodinámica. Universidad de las Fuerzas Armadas ESPE. Energía y Mecánica.

NASA. (8 de enero de 2017). NASA Surface meteorology and Solar Energy: RETScreen Data. Obtenido de NASA Surface meteorology and Solar Energy: RETScreen Data.: https://eosweb.larc.nasa.gov/cgi$\mathrm{bin} / \mathrm{sse} /$ retscreen. cgi?email=\&step $=1 \&$ lat $=10.435978 \&$ lon $=-$ 73.265226\&submit=Submit

POZOS SUÁREZ, I. (2010). DISENO TÉRMICO PARA UN HORNO SOLAR DE SECADO DE MADERA. Xalapa-Eqz.

RAG León, EF Solano. (2017), Análisis Estructural De Unamáquina Prensadora Para Producción De Ladrillo Macizo Para Las Pequeñas Industrias Artesanales ... Revista Colombiana de Tecnologías de Avanzada ISSN: 1692-7257.

universidad de madrid. (3 de mayo de 2017). transformacion natural de la energia solar. Obtenido de http://www.educa.madrid.org/web/ies.vict oriakent.torrejondeardoz/Departamentos/ DFyQ/energia/e-3/energia2.htm 